Z Gerontol Geriat

DOI 10.1007/s00391-016-1135-9

(c) Springer-Verlag Berlin Heidelberg 2016

CrossMark

Eva Schulc ${ }^{1}$ Martin Pallauf' $\cdot$ Tanja Wildbahner ${ }^{2} \cdot$ Christa Them $^{2}$

'Institut für Gerontologie und demographische Entwicklung, UMIT - Private Universität für Gesundheitswissenschaften, Medizinische Informatik und Technik, Hall in Tirol, Österreich ${ }^{2}$ Institut für Pflegewissenschaft, UMIT - Private Universität für Gesundheitswissenschaften, Medizinische Informatik und Technik, Hall in Tirol, Österreich

\title{
Erratum zu: Präventive Hausbesuche
}

\section{Querschnittstudie zur Unterstützung der selbstständigen Lebensführung älterer Menschen}

\section{Erratum zu: \\ Z Gerontol Geriat (2016) \\ 49:526}

In der veröffentlichten Version des Beitrags wurde eine falsche Reihenfolge der Autoren angezeigt.

Der Verlag bittet, den Fehler zu entschuldigen und um Beachtung der oben aufgeführten korrekten Version.

\section{Korrespondenzadresse}

\section{MMag. Dr. E. Schulc}

Institut für Gerontologie und demographische Entwicklung, UMIT - Private Universität für Gesundheitswissenschaften, Medizinische Informatik und Technik

Eduard-Wallnöfer-Zentrum 1,6060 Hall in Tirol, Österreich

eva.schulc@umit.at 